\title{
Computed Tomography-based Patient-specific Biomechanical and Fluid Dynamic Study of Anomalous Coronary Arteries with Origin from the Opposite Sinus and Intramural Course
}

\author{
Gianluca Rigatelli1 and Marco Zuin ${ }^{1,2}$ \\ 1. Cardiovascular Diagnosis and Endoluminal Interventions Unit, Rovigo General Hospital, Rovigo, Italy; 2. Section of Internal and \\ Cardiopulmonary Medicine, University of Ferrara, Ferrara, Italy
}

DOI: https://doi.org/10.17925/HI.2020.14.2.105

\begin{abstract}
$\mathrm{B}$ ackground: The anomalous coronary arteries originating from the opposite sinus of Valsalva (ACAOS) constitutes one of the most clinically relevant coronary artery anomalies in adults. Exact pathophysiology and the impact of intramural (IM) course segment stenting in ACAOS with IM course (ACAOS-IM) has not been clarified. We aimed to elucidate the pathophysiology and impact of stenting applying biomechanical and computational fluid dynamics to computed tomography (CT) in patient-specific coronary vessel reconstruction. Methods: We separated coronary artery (left or L-, right or R-) ACAOS-IM into segments (proximal, mid and distal), based on coronary angiography and coronary CT angiography features, in a series of patients at Rovigo General Hospital, Italy, between 1 January 2003 and 1 January 2018. Blood pressure gradient across the coronary circulation, calculated blood flow, vorticity magnitude, wall shear stress (WSS) and IM segment deformation were analysed by simulating exercise, before and after virtual stent implantation. Results: In 21 symptomatic patients (13 males, mean age 46.1 \pm 8.1 years, L-ACAOS-IM in 9 and R-ACAOS-IM in 12 patientS), computational fluid dynamic analysis in both L- and R-ACAOS demonstrated higher basal WSS values in the IM course $(9.5 \pm 0.2$ and $8.6 \pm 0.2$ Pa for R- and L-ACAOS, respectively), than in the rest of the vessels. These values decreased after stenting. Vorticity magnitude significantly decreased after stenting as well, compared with baseline. Biomechanical deformation analysis revealed not only compression, but also a twisting of the IM segment with a mean distal pressure drop of 32\% and 35\% in R- and L-ACAOS, respectively, which was corrected by stent implantation. Conclusions: In both L- and R-ACAOS subtypes, the IM segment appeared to be phasically compressed and deformed with a degree of twisting that causes resting and exercise cross-sectional deformation and a drop in distal pressure. Stenting of the IM segment results in normalisation of the flow profile, correction of the IM segment deformation and reverses the drop in pressure, for both variants of ACAOS.
\end{abstract}

\section{Keywords}

Coronary artery anomalies, sudden death, physiology, computed fluid dynamic

Disclosures: Gianluca Rigatelli and Marco Zuin have no financial or non-financial relationships or activities to declare in relation to this article.

Review Process: Double-blind peer review.

Compliance with Ethics: All procedures were followed in accordance with the responsible committee on human experimentation and with the Declaration of Helsinki of 1975 and subsequent revisions, and informed consent was received from all patients involved in this study. Authorship: The named authors meet the criteria of the International Committee of Medical Journal Editors for authorship for this manuscript, take responsibility for the integrity of the work as a whole and have given final approval for the version to be published.

Access: This article is freely accessible at touchCARDIO.com (c) Touch Medical Media 2020.

Received: 10 September 2020

Accepted: 4 December 2020

Published Online: 23 December 2020

Citation: Heart International. 2020;14(2):105-11 Corresponding Author: Gianluca Rigatelli, MD, PhD, EBIR, Cardiovascular Diagnosis and Endoluminal Interventions, Santa Maria della Misericordia Hospital, Viale Tre Martiri 140, 45100 Rovigo, Italy

E: jackyheart@libero.it

Support: No funding was received for

the publication of this article.
Anomalous coronary arteries originating from the opposite sinus of Valsalva (ACAOS) is one of the most clinically relevant abnormalities among the wide spectrum of coronary artery anomalies. ${ }^{1}$ sudden cardiac death has been related to myocardial bridges, ectopic origin of the pulmonary artery, a single coronary artery and ACAOS with an intramural course (ACAOS-IM), especially in young athletes. ${ }^{2-4}$ Anatomically, the IM segment is embedded into the aortic tunica media where elastic tissue is present, but smooth cells are absent. Both in athletes and patients from the general population who have an ACAOS-IM, myocardial ischaemia seems to be caused by a phasic compression of the IM course, as demonstrated by intravascular ultrasonography (IVUS). ${ }^{5}$ Unfortunately, both non-invasive stress tests and fractional flow reserve (FFR) have failed to objectively confirm the causality relationship (compression-ischaemia) in the majority of individuals with such an anomaly, ${ }^{6}$ predominantly because it is difficult to recreate the real conditions in which IM compression occurs. As result, the specific blood flow mechanisms underlying myocardial ischaemia in both right (R)- and left (L)-ACAOS-IM variants have not yet been adequately clarified and the indication for interventional treatment continues to be interrogated.

In medicine, computational fluid dynamics (CFD) is the off-line study of the fluid stream within vessels and chambers, computationally reconstructed utilising data from in vivo imaging investigations. ${ }^{7}$ In recent years, CFD has gained a reputation in the evaluation of pathophysiology and therapeutic interventions that are difficult to assess in vivo, and a recent study into CFD confirmed that in L-ACAOS, the IM course is compressed when a sufficient amount of intra-aortic blood pressure is input into the computerised model. ${ }^{8}$

In the present study, we sought to further elucidate the underling pathophysiological mechanism of ischaemia in both L-ACAOS-IM and R-ACAOS-IM, as well as to evaluate the impact of IM segment stenting, using CFD analysis. 


\section{Materials and methods Population}

The study group consisted of symptomatic patients with ACAOS who underwent catheter coronary angiography in at Rovigo General Hospital, Rovigo, Italy between 1 January 2003 and 1 January 2018. As per the institutional protocol, which was approved by our Institutional Review Board, these patients were assessed with a uniform multi-step diagnostic work-up, including a cycle ergometer stress test, transthoracic echocardiography, catheter coronary angiography (IVUS was eventually used at the physician's discretion to confirm IM course in cases of uncertainty), and coronary computed tomography angiography (CCTA). Patients with ACAOS who had undergone catheter coronary angiography for heart failure or valvular heart disease (mitral valve and/or aortic valve stenosis, or insufficiency greater than moderate) and patients with evidence of coronary artery disease alongside ACAOS identified as having at least one significant coronary artery stenosis with luminal narrowing $>70 \%$ by quantitative coronary angiography, were excluded from the study.

\section{Coronary angiography protocol}

Coronary angiography was performed using a 6F radial approach whenever possible, or alternatively, a 4F sheath and catheters were used through the femoral artery. A Tilon bilateral diagnostic catheter (Johnson \& Johnson, New Brunswick, NJ, USA) or Amplatz left (1 or 2) diagnostic catheters (Amplatz Cordis Europe, Roden, The Netherlands) were usually used to cannulate selectively the right ostium from the left sinus of Valsalva, while an Amplatz Left curve, a 6F Ebu or Champ guiding catheter (Medtronic Inc., Minneapolis, MN, USA) were used to cannulate the left ostium of the right sinus. The $3 \mathrm{~F}_{\text {OpticrosS }}{ }^{\mathrm{TM}}$ coronary IVUS catheter (Boston Scientific, Fremont, CA, USA) and automatic pull-back system $(0.5 \mathrm{~mm} / \mathrm{sec})$, was used for IVUS examination, as described by Angelini et al. ${ }^{9}$

\section{Coronary computed tomography angiography protocol}

CCTA images were acquired using a 64-slice multi-detector CT scanner (64-detector row LightSpeed VCT scanner [GE Healthcare, Milwaukee, WI, USA]). Before the imaging acquisition, patients were pre-treated with oral and/or intravenous beta-blockers, as necessary, to achieve a target resting heart rate of $<70$ beats per minute. Electrocardiographic gating was used for all scans and image acquisition was gated in the diastolic phase. The scanning protocol was adjusted for patient weight and heart rate. A bolus intravenous dose of iodine contrast was given by mechanical injection through the basilic or cephalic vein. The entire volume of the heart was measured in 8-9 seconds during a single breath-hold. Each examination was independently interpreted by a trained cardio-radiologist using a combination of axial images, 3D volume-rendered images, and multiplanar reformations.

\section{Model reconstruction and analysis}

Coronary artery circulation was reconstructed for each patient using the morphologic data obtained by quantitative CCTA using the Osirix software (OsiriX Foundation, Geneva, Switzerland). The model was subsequently optimsed using the Rhinoceros evaluation software, version 4.0 (McNeel \& Associates, Indianapolis, IN, USA), as previously described. ${ }^{10}$ Blood was modelled as a non-Newtonian, viscous and incompressible fluid. Density was defined as 1,060 kg/m³ according to the standard literature. ${ }^{11}$ Blood was represented by the Navier-Stokes and continuity equations. ${ }^{12}$

A Carreau model was adopted to simulate viscosity. ${ }^{13}$ Because coronary artery perfusion occurred primarily in diastole, at least in the left coronary
Figure 1: Schematic representation of the biomechanics of twisting, applied to our model

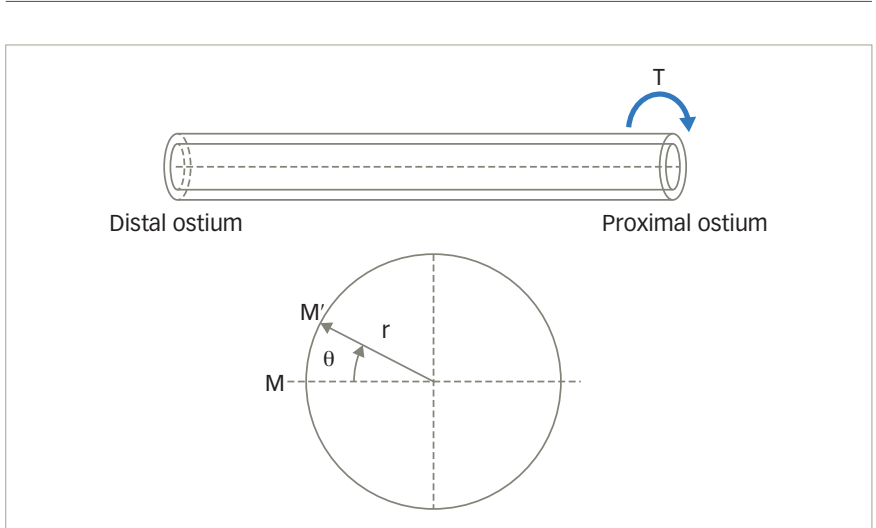

The computational parameter, also known as longitudinal deformation, was measured as the angle of twist between the two centroids at $25 \%, 50 \%$ and $75 \%$ of the intramural segment, respectively.

$\theta=$ angle of twist; $M=$ moment of torsion; $r$ = radius; $T$ = torque.

artery, at the inlet of coronary ostia, we considered a steady blood flow ${ }^{14}$ and a pressure peak value retrieved from the patient-specific ergometric stress tests. Using an ergometric test with sport protocol (increment of $50 \mathrm{~W}$ every 1 minute), the diastolic and systolic blood pressures at the $85 \%$ of the maximal age-predicted heart rate $(110.8 \pm 9.1$ and $190.4 \pm 10.4 \mathrm{mmHg}$, respectively) were used as pressure peak values.

\section{Vorticity magnitude, shear stress and deformation and torsion}

The vorticity magnitude was defined as the magnitude of the vorticity vector, while using vorticity as a measure of the blood rotation into the vascular domain. Conversely, wall shear stress (WSS) (measured in Pa) was defined as the force which tangentially acted on the inner vascular surface, due to friction. ${ }^{15}$

The IM tract deformation, which is defined as any geometrical (axial and radial) changes of the IM segment in terms of movements and/or distortions, was evaluated in terms of axial length and curvature change. Specifically, the artery length change was quantified by calculating the difference between the length of the centerline artery in the pre- and post- stenting configurations. Conversely, the curvature change in the IM tract was assessed at multiple points (25\%, $50 \%$ and $75 \%$ of the length of the IM segment) and compared to the curvature values at the same measurement position in the pre-stenting configuration. The elastic deformation was expressed in $\mathrm{mm}$ in both length and diameter in both pre- and post-stenting configurations.

As demonstrated for other coronary districts, such as bifurcations ${ }^{16}$ and as has been shown with anatomy specimens, ${ }^{17}$ the IM segment is fixed in correspondence with the two ostia: the former is the opposite sinus ostium while the latter is the 'real' ostium at the exit of the IM course. The two ostia represent two fixed binding points at either end of the IM tract, which generate a resisting force. The torsion of an elastic pipe (e.g. the IM segment, because of the elastic return caused by the systolic phase) creates both distortion and loss of energy, worsening the mechanical properties and the rheology of the flowing fluid. The parameters involved in a torsion, or twisting, are the torque $(T)$, the diameter $\left(r^{2}\right)$ of the shaft and the angle of twist (defined as the angle $\theta$ generated by the displacement of $\mathrm{M}$ to $\mathrm{M}^{\prime}$ ) (Figure 1).

To analyse the torsion, we set a local reference system. The $x$ axis has been defined as the line passing through the centroids of two 
Figure 2: Computational appearance of reconstructed Orsiro drug-eluting stent

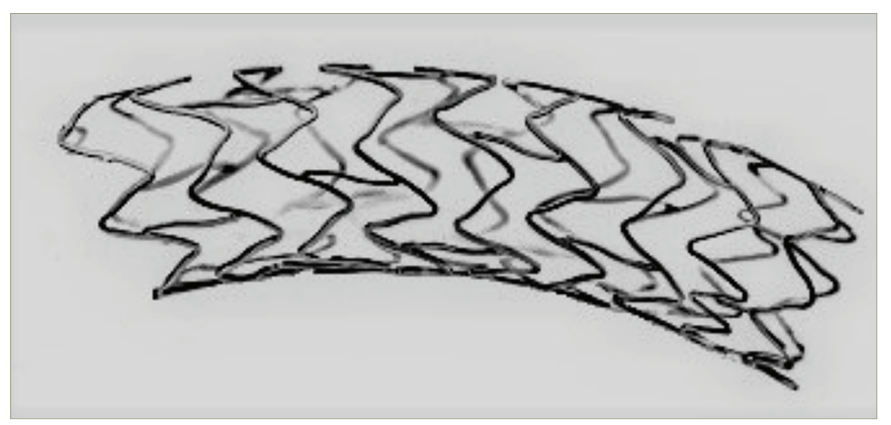

cross-sectional areas at $25 \%$ and $75 \%$ of the IM tract length, respectively. The $z$ axis was set perpendicular to the $x-y$ reference plane and point anteriorly (out of the page), following the right-hand rule. Moreover, the two pilot nodes have been set in correspondence of the two ostia. The drop of pressure in Pa due the IM course, was converted to $\mathrm{mmHg}$, because interventional cardiologists are more familiar with this measurement unit.

\section{Stent geometry reconstruction}

For the stent simulation, we reconstructed the strut design and linkage pattern of a third-generation everolimus-eluting stent (Orsiro stent [Biotronik IC, Bulack, Switzerland]), commonly used in our institution. The strut thickness of this stent is characterised by a $60 \mu \mathrm{m}$ for $3.0 \mathrm{~mm}$ diameter stent and $80 \mu \mathrm{m}$ for $4.0 \mathrm{~mm}$ stent. Computer-aided design software was used to reproduce the stented geometry as accurately as possible (SOLIDWORKS ${ }^{\circledR} 2009$ [Solidworks Corp., Concord, MA, USA]). For the first step, we created the solid model of the ACAOS and then the deployed stent geometry (Figure 2). For this purpose, a hollow tube with an outer diameter equal to both the nominal expanded diameter and thickness of the stent was created. Then, a two-dimensional sketch with the stent's strut was propagated and wrapped around the tube. Through a cut out, the obtained ring of the stent was propagated axially to create the full-length expanded model.

\section{Virtual implantation}

After placing the stent model in the correct position, the stenting procedure was simulated on the basis of the following procedural steps. Stent diameter and length were chosen based on quantitative coronary angiography and CCTA mean measurements.

- Direct stent implantation: Orsiro $4.0 \times 15 \mathrm{~mm}$ at $18 \mathrm{~atm}$ for L-ACAOS and $3.5 \times 15 \mathrm{~mm}$ at $18 \mathrm{~atm}$ for R-ACAOS.

- Post-dilation with $4.5 \times 15 \mathrm{~mm}$ for L-ACAOS and $3.75 \times 15 \mathrm{~mm}$ Noncompliant EuphoraTM (Medtronic Inc., Minneapolis, MN, USA) balloon at $20 \mathrm{~atm}$ for R-ACAOS.

Using the Boolean operation, the modified solid model was subtracted from the model to obtain the final geometry. We assumed that, after stent deployment and implantation, there was no residual stenosis or as little as possible. The numeric grid was created from the geometry using Ansys Meshing 14.0 (Ansys, Inc., Canonsburg, PA, USA) while the simulations were conducted using the commercial software Ansys Fluent 14.0 (Ansys, Inc., Canonsburg, PA, USA).

\section{Statistical analysis}

For each group (R- and L-ACAOS-IM), continuous variables were expressed as mean \pm standard deviation (SD) and were compared by Student's t-test if the data had normal distribution, otherwise values
Table 1: Demographic and clinical data of the collected patients used for the model reconstruction and computational fluid dynamics analysis

\begin{tabular}{|l|l|}
\hline Demographic data & $46.1 \pm 8.1$ \\
\hline Age (years \pm SD) & $13(61.9)$ \\
\hline Male gender, $n$ (\%) & $7(33.3)$ \\
\hline Hypercholesterolemia, $n$ (\%) & $4(19.0)$ \\
\hline Hypertension, $n$ (\%) & $0(0)$ \\
\hline Diabetes, $n$ (\%) & $2(9.5)$ \\
\hline Heart valve disease, $n$ (\%) & \\
\hline Indications for coronary angiography & $8(38.1)$ \\
\hline Atypical chest pain during effort, $n$ (\%) & $6(28.6)$ \\
\hline Unstable angina, $n$ (\%) & $3(14.3)$ \\
\hline $\begin{array}{l}\text { NSTEMI, } n \text { (\%) } \\
\text { Cardiac arrest, } n \text { (\%) } \\
\text { Sustained ventricular tachyarrhythmias, } n(\%)\end{array}$ & $1(4.7)$ \\
\hline On 24-hour Holter, $n$ (\%) & $7(33.3)$ \\
\hline During stress test, $n$ (\%) & $5(23.8)$ \\
\hline Positive Stress test (bicycle-ergometric test) & $2(9.5)$ \\
\hline Angiographic findings other than CAA & $4(19.0)$ \\
\hline Superimposed CAD on ACAOS, $n$ (\%) & \\
\hline Associated CAD on other coronary vessels, $n$ (\%) & $6(0)$ \\
\hline
\end{tabular}

ACAOS = anomalous coronary arteries originating from the opposite sinus of Valsalva; $C A A=$ coronary artery anomaly; $C A D=$ coronary artery disease, defined as at least one significant coronary artery stenosis $>50 \%$ luminal narrowing; NSTEMI = nOn-ST segment elevation myocardial infarction, as defined by Thigesen et al. ${ }^{24}$

SD = standard deviation

Table 2: Results of computational fluid dynamic analysis

\begin{tabular}{|c|c|c|c|}
\hline & Pre-stent & Post-stent & p-value \\
\hline \multicolumn{4}{|l|}{ L-ACAOS $(n=9)$} \\
\hline $\begin{array}{l}\text { WSS }_{\text {intramural }}(\mathrm{Pa}) \text {, mean } \\
\pm \mathrm{SD}\end{array}$ & $9.5 \pm 0.2$ & $6.8 \pm 0.1$ & $<0.001$ \\
\hline Mean WSS RCA & $5.3 \pm 1.1$ & $4.7 \pm 0.7$ & 0.24 \\
\hline Mean WSS LCA & $5.1 \pm 0.4$ & $3.8 \pm 1.7$ & 0.72 \\
\hline Mean VM (1/s) & $9,008.26$ & $6,012.02$ & $<0.001$ \\
\hline \multicolumn{4}{|l|}{ R-ACAOS $(n=12)$} \\
\hline $\begin{array}{l}\text { WSS }_{\text {intramural }}(\mathrm{Pa}), \text { mean } \\
\pm \mathrm{SD}\end{array}$ & $8.6 \pm 0.2$ & $5.2 \pm 0.3$ & $<0.001$ \\
\hline Mean WSS RCA & $5.8 \pm 1.1$ & $4.9 \pm 1.3$ & 0.18 \\
\hline Mean WSS LCA & $5.0 \pm 0.8$ & $4.8 \pm 0.8$ & 0.64 \\
\hline Mean VM (1/s) & $8,926.15$ & $6,021.42$ & $<0.001$ \\
\hline
\end{tabular}

Vorticity magnitude was defined as the measure of the rotation of blood in the flow field per second.

ACAOS = anomalous coronary arteries originating from the opposite sinus of Valsalva; $L=$ left; $L C A=$ left coronary artery; $R=$ right; $R C A$ = right coronary artery; $V M=$ vorticity magnitude; $W S S=$ wall shear stress forces .

were compared using the Wilcoxon-Mann-Whitney U test. Categorical variables were presented as proportions and compared using the Pearson's $\chi^{2}$ test. All statistical analyses were carried out using SPSS statistical software version 19.0 (SPSS Inc., Chicago, IL, USA); $p<0.05$ was considered statistically significant.

\section{Results}

A total of 21 symptomatic patients with ACAOS were included in the study; 13 males, mean age $46.1 \pm 8.1$ years. Nine patients had L-ACAOS and 12 had R-ACAOS. Clinical presentation and indications for coronary angiography are shown in Table 1. 

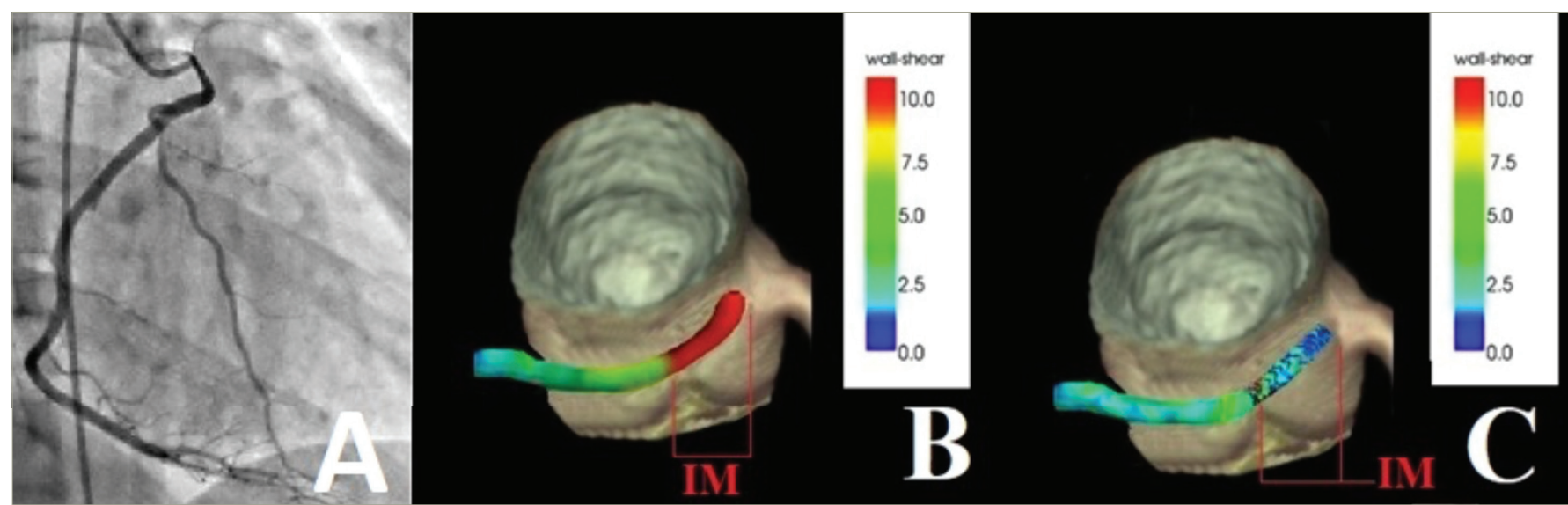

Wall shear stress in a 41-year-old man with R-ACAOS-IM (A), the normalisation of WSS after stenting $(B)$ the IM course (from red to green-blue colour), and 3D reconstruction of the IM tract (C).

$I M=$ intramural; $R$-ACAOS-IM = right anomalous coronary arteries originating from the opposite sinus of Valsalva with an intramural course; WSS = wall shear stress

Figure 4: Wall shear stress in a 41-year-old man with L-ACAOS-IM

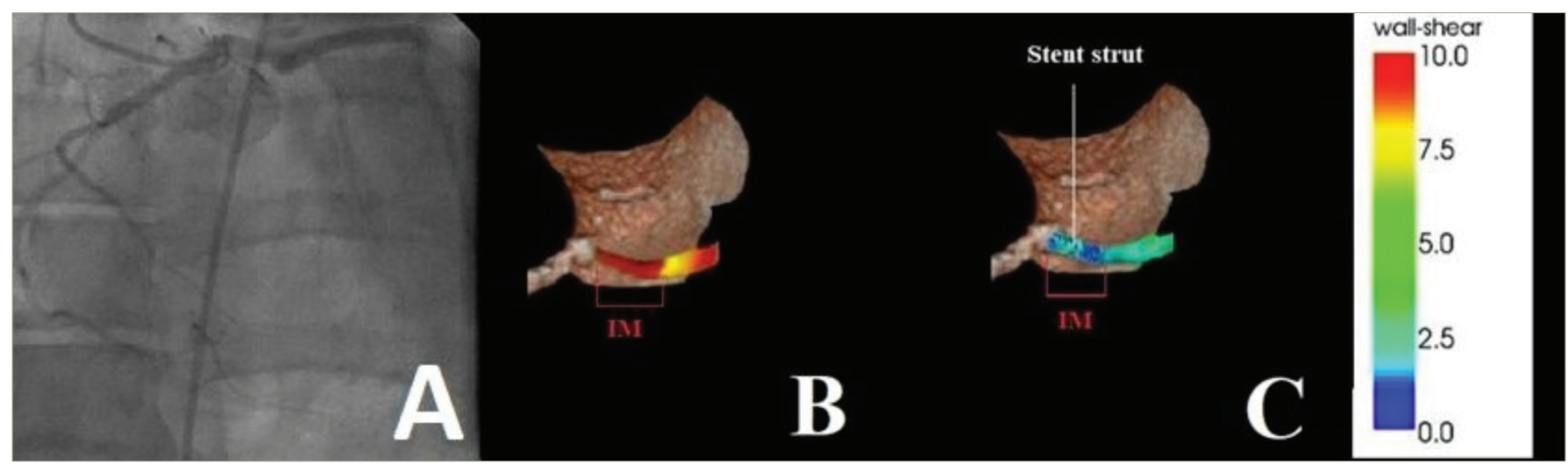

(A) Wall shear stress representation Note the normalisation of WSS after stenting (B) of the IM course (from red to green-blue colour). 3D-reconstruction of the IM tract (C). $I M=$ intramural; L-ACAOS-IM = left anomalous coronary arteries originating from the opposite sinus of Valsalva with an intramural course; WSS = wall shear stress.

Wall shear stress and vorticity magnitude findings CFD analysis demonstrated higher basal WSS values in the IM course $\left(\right.$ WSS $\left._{\text {IM }}\right)(9.5 \pm 0.2$ and $8.6 \pm 0.2 \mathrm{~Pa}$ for R- and L-ACAOS, respectively). The mean WSS $_{\mathrm{IM}}$ significantly decreased after virtual stent implantation $(9.5 \pm 0.2 \mathrm{~Pa}$ versus $6.8 \pm 0.1 \mathrm{~Pa}, \mathrm{p}<0.001$; and $8.6 \pm 0.2 \mathrm{~Pa}$ versus $5.2 \pm 0.3 \mathrm{~Pa}, \mathrm{p}<0.001$; for $\mathrm{R}$ - and L-ACAOS respectively) resulting in value normalisation (Table 2, Figure 3 and Figure 4). Conversely, no significant reduction in the mean WSS of the distal right and left coronary arteries was observed after the stenting. Similarly, the vorticity magnitude significantly also decreased compared to the baseline pattern, after stenting in both L- and R-ACAOS (Table 2). Pre- and post-stenting comparison of the twisting angle in the IM segment is shown in Table 3.

\section{Elastic deformation, torsion and relationship with hydrostatic pressure}

Deformation analysis was applied to the IM segment which had a length of $7.4 \pm 2.7 \mathrm{~mm}$ and $8.1 \pm 2.2 \mathrm{~mm}$ in R-ACAOS and L-ACAOS patients, respectively. A lower axial and curvature change of the IM segment during the simulated exercise was apparent before and after stenting (Figure 5), with a consistent decrease in the cross-sectional area of the IM segment (Figure 6) in both R- and L-ACAOS-IM. Before stenting, hydrostatic pressure value was higher at the entry compared with the exit of IM segment with a reduction of
Table 3: Pre- and post-stenting comparison of the twisting angle in the intramural segment

\begin{tabular}{|l|l|l|l|}
\hline & $\begin{array}{l}\text { Pre-stent angle } \\
\text { degree }\end{array}$ & $\begin{array}{l}\text { Post-stent angle } \\
\text { degree }\end{array}$ & p-value \\
\hline R-ACAOS & $14.3^{\circ} \pm 2.3^{\circ}$ & $7.5^{\circ} \pm 1.8^{\circ}$ & $p<0.001$ \\
\hline L-ACAOS & $6.2^{\circ} \pm 1.2^{\circ}$ & $13.7^{\circ} \pm 1.8^{\circ}$ & $p<0.001$ \\
\hline
\end{tabular}

ACAOS = anomalous coronary arteries originating from the opposite sinus of Valsalva; $L=$ left; $R=$ right.

$>30 \%$ in both R- and L-ACAOS; this gradient was diminished after stenting (Figure 7).

A significant negative correlation between the axial twisting and the length of the IM tract was observed in unstented $\mathrm{R}-(\mathrm{r}=-0.433, \mathrm{p}=0.030)$ and L-ACAOS ( $r=-0.430, p=0.032$ ) patients, respectively (Figure 8). Moreover, axial twist angle significantly decreased after stenting the IM tract.

\section{Discussion}

Our study suggests that the pathophysiological mechanism of ischaemia in both R- and L-ACAOS might be more complex than the old interarterial course concept. While our results showed compression of the IM segment, a degree of twisting of the IM course also seemed to contribute 
Figure 5: Analysis of geometrical deformation

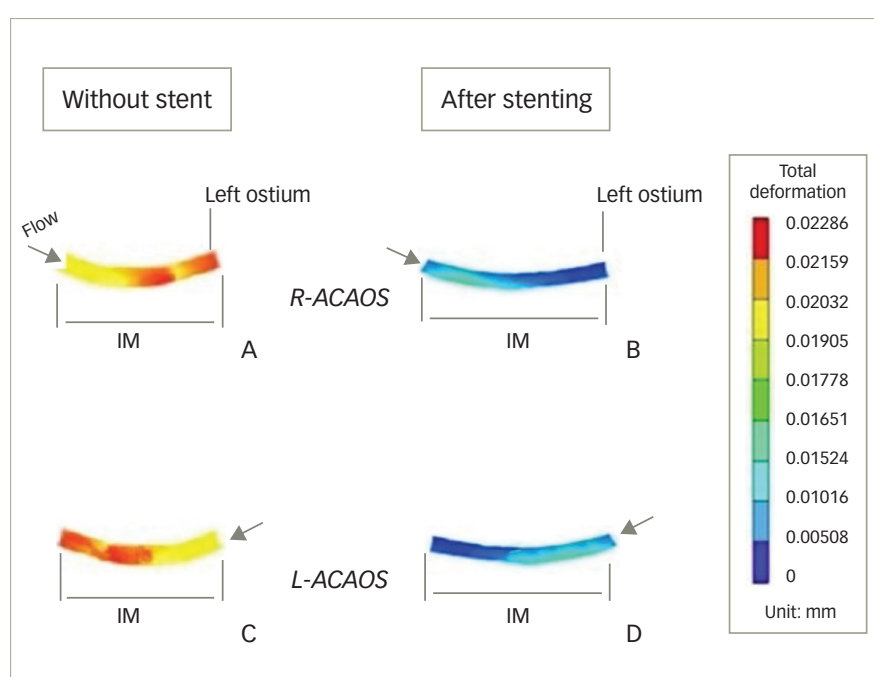

Before and after stenting within the IM segment in two patients with R-ACAOS $(A, B)$ and $L-A C A O S(C, D)$. Note the reduced deformation after stenting (from red to green-blue color).

ACAOS = anomalous coronary arteries originating from the opposite sinus of valsalva; $I M=$ intramural; $L=$ left; $R=$ right.

Figure 6: Mean systolic and diastolic cross-sectional deformation

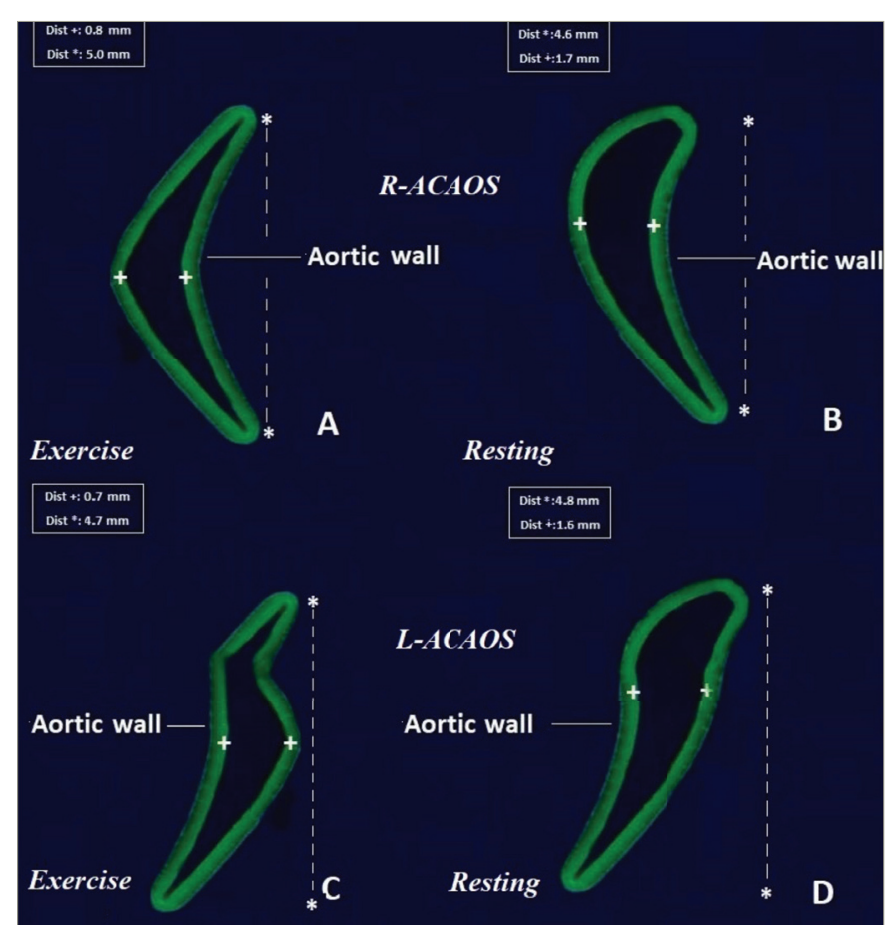

Patients with R-ACAOS-IM (A, B) and patients with L-ACAOS-IM (C, D). Images obtained from the CFD analysis of vessels $3 D$ reconstruction obtained from a signal-averaged image of enrolled patients.

* Vertical distance in milimetres.

+ Horizontal distance in milimetres.

ACAOS = anomalous coronary arteries originating from the opposite sinus of Valsalva; $C F D=$ computational fluid dynamic; Dist. = distance; $L=$ left; $R=$ right.

in the compression of the segment, further reducing perfusion pressure distally to the IM segment. Moreover, our analysis outlines that an eventual stenting of the IM segment would drastically reverse this compression and normalise the flow pattern profile in both ACAOS variants, avoiding at the same time the vessel squeezing and increasing the distal perfusion pressure.
Figure 7: Mean diastolic pressure at entry and exit ostia of the intramural segment before and after stenting
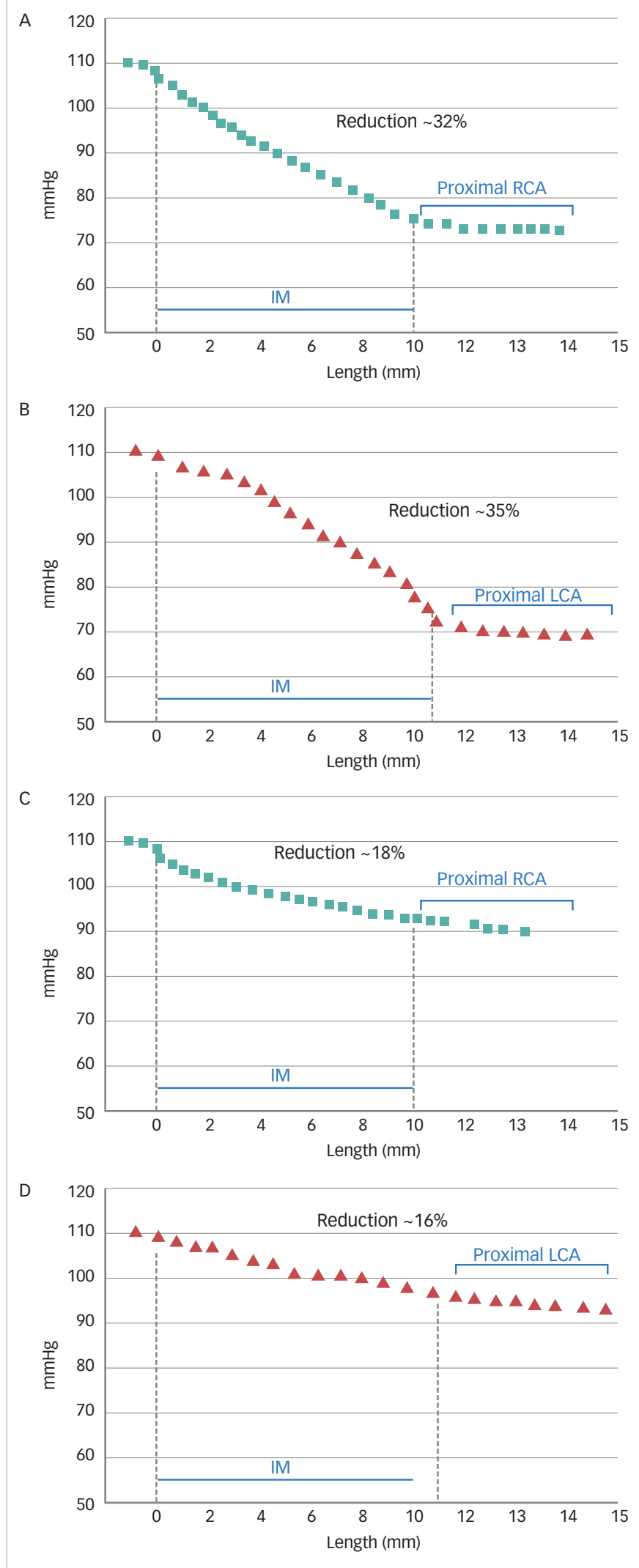

Mean diastolic pressure at entry $(A)$ and exit (B) ostia of the IM segment before stenting. Mean diastolic pressure at entry $(C)$ and exit (D) ostia after stenting. $R$-ACAOS-IM $(A-C)$, L-ACAOS-IM $(B-D)$. Note the pressure drop $(A, B)$ and its relief after stenting $(C, D)$ in each anomaly model. Each symbol represents three iterations generated during the analysis. Green square: diastolic pressure at entry ostia. Red triangle: diastolic pressure at exit ostia.

ACAOS = anomalous coronary arteries originating from the opposite sinus of Valsalva; $I M=$ intramural; $L=$ left; $L C A=$ left coronary artery; $R=$ right; $R C A=$ right coronary artery. 
Figure 8: Correlation between axial twisting angle and length of the intramural segment

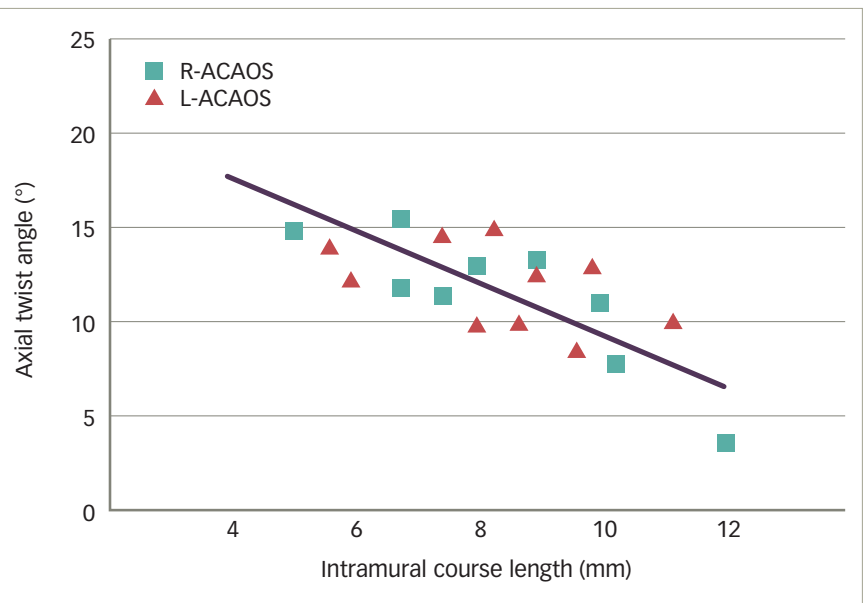

ACAOS = anomalous coronary arteries originating from the opposite sinus of valsalva; $L=$ left; $R=$ right.

As previously demonstrated, ${ }^{18}$ a phasic stenosis that can be detected with IVUS imaging, especially when using dobutamine as a stress-simulating agent (as showed by Angelini et al. ${ }^{19}$ ), is not able to completely explain myocardial ischaemia alone in these patients. Despite the evidence from previous research in phasic stenosis by IVUS, ${ }^{19,20}$ the noninvasive tests proposed to investigate the clinical significance of ACAOS-IM, and more recently, physiologic invasive tests, such as FFR, ${ }^{6}$ were not able to give real prognostication and were positive only in the minority of patients. The 'strenuous exercise' that has been associated with sudden cardiac death in athletes and military recruits seems not only the fundamental modifier, but also the reason why the stress test and the invasive measurements were not able to identify a participant at risk. Strenuous exercise is probably the most difficult condition to reproduce.

Our analysis showed an amount of vorticity and longitudinal deformation of the IM segment very similar to what is usually called a 'twisting', which is usually not a clinical issue in normal coronary segments, but when associated with lateral compression, it might exacerbate reduction of cross-sectional area. Although this would be a computational observation rather than clinical instrumental proof, it appears clear that the combination of compression and twisting may reduce the IM luminal area enough to produce a consistent pressure drop with distal reduction of the perfusion. As observed in our study, the length of the IM segment would also be of a certain importance, due the existence of a negative correlation between the axial twisting and the length of the IM tract.

It also appears likely that the length of IM segment could be correlated with the amount of compression and twisting, leading to a more clinically evident pressure drop as the length increases in different individuals. Although not replicated by further studies, in 2011 Kaushal et al. suggested that the length of the IM segment was a determinant of the symptoms and severity of clinical presentation. ${ }^{21}$

Our hypothesis is that in particular extreme and stressful conditions, an increase in intra-aortic blood pressure sustained for minutes is able to compress and twist the long IM segment sufficiently to produce a clinically evident pressure drop distally to the IM segment. After stenting, a mean gradient remained, generating a significant haemodynamic pressure drop. The reasons for this could be multifactorial and a result of some residual anatomical high-risk features, such as slit-like ostium, acute take-off angle and proximal narrowing and elliptic vessel shape.

Over the pastyears, the management ofACAOS-IM was based essentially on good clinical sense more than on guideline recommendations. Once myocardial ischaemia is found, which can be through non-invasive tests in rare instances, or, more frequently, using IVUS, therapeutic options range from stenting to open heart surgery, typically by coronary un-roofing. Data on clinical effectiveness and the long-term impact of such treatments are obviously still lacking due to the rarity of the anomaly, the difficult diagnosis and worldwide heterogeneous management. Recently, it has been suggested that surgical repair of ACAOS does not completely eliminate the risk of sudden cardiac death at a mean follow up of 1.6 years. ${ }^{22}$

Although classical surgical repair is readily accepted as a definitive treatment, the efficacy of IM segment stenting is far to be proven. Our computational investigation confirmed the previously demonstrated clinical benefit of stenting, in terms of the IM fluid profile, eliminating compression, and near abolishment of the twisting. Whether this strategy would be clinically effective obviously is beyond the scope of the study and requires further investigation in complex and difficult-to-conceive clinical studies. In any case, our virtual computational observations give further positive rheologic confirmation to the anecdotal cases and series treated by stenting that have been reported in literature.23,24

\section{Limitations}

Our study suffers from several limitations. Firstly, it utilises a virtual $\mathrm{R}$ - and L-ACAOS model and the vessel has been considered non-compliant with a steady diastolic blood flow in a virtual haemodynamically stable patient; previous studies have already demonstrated that myocardial motion has a negligible effect on blood flow distribution of the coronary tree. ${ }^{8,15}$ Secondly, we investigated our model only in the diastolic phase, while the phasic obstruction in ACAOS-IM is thought to occur during cardiac systole. However, coronary artery perfusion is mainly diastolic and, so as not to diminish the effect of myocardial motion, we felt the diastolic phase was more reliable. We did not evaluate the time-averaged WSS, oscillatory index, and the relative residence time, which play a recognised role in the development of arterial atherosclerosis, but have been judged less important in studying a phasic phenomenon.

We used CFD analysis instead of FFR because previous investigations have already demonstrated that steady-state CFD simulation represents a more practical approach given that it requires much less computational time. Moreover, FFR has been designed to analyse a 'steady' and not a dynamic or better phasic coronary artery occlusion, as in the case of $\mathrm{R}$ - or L-ACAOS. In such anomalies, there is a cross-sectional decrease of the IM segment in rest (also called proximal hypoplasia), which is even more pronounced during physical exercise as a result of the lateral compression. This decrease, however, affects both systole and diastole, causing a significant reduction in coronary perfusion, which has well-known consequences.

\section{Conclusion}

Our CFD-based investigation suggests that not only compression but also twisting of the IM course would occur under exercise conditions reducing blood flow distally to the IM segment and potentially causing myocardial 
ischaemia and malignant arrhythmias in individuals with L- and R-ACAOS with IM course. Length of the IM segment seems to correlate to the amount of twisting and IM segment deformation. Stenting with a modern third-generation drug-eluting stent would potentially normalise the flow patterns in terms of WSS and vorticity, reducing the twisting in patients with R-ACAOS but increasing it in those with L-ACAOS. $\square$
1. Rigatelli $\mathrm{G}$, Rigatelli $\mathrm{A}$, Cominato $\mathrm{S}$, et al. A clinical-angiographic risk scoring system for coronary artery anomalies.

2. Peterson DF Siebert DM, Kucera KL, et al. Etiology of sudden cardiac arrest and death in US competitive athletes: a 2-year prospective surveillance study. Clin I Sport Med. 2020;30:305-14

3. Angelini P, Cheong BY, Lenge De Rosen W, et al. High-risk cardiovascular conditions in sports-related sudden death: prevalence in 5,169 schoolchildren screened via cardiac magnetic resonance. Tex Heart Inst J. 2018;45:205-13.

4. Pawlowicz B, Fernandes J, Nair V. Coronary artery abnormalities as the cause of sudden cardiac death: a 20-year review. Am J Forensic Med Pathol. 2018;39:114-8.

5. Angelini P, Uribe C. Anatomic spectrum of left coronary artery anomalies and associated mechanisms of coronary
insufficiency. Catheter Cardiovasc Interv. 2018;92:313-21.

6. Driesen BW, Warmerdam EG, Sieswerda GT, et al. Anomalous coronary artery originating from the opposite sinus of Valsalva (ACAOS), fractional flow reserve- and intravascular ultrasound-guided management in adult patients. Catheter Cardiovasc Interv. 2018;92:68-75.

7. Zuin M, Rigatelli G, Faggian G, Roncon L. Mathematics and cardiovascular interventions: role of the finite element modeling in clinical decision making. JACC Cardiovasc Interv. 2016;9:507-8

8. Rigatelli G, Zuin M, Galasso P, et al. Mechanisms of myocardia ischemia inducing sudden cardiac death in athletes with anomalous coronary origin from the opposite sinus: Insights from a computational fluid dynamic study. Cardiovasc Revasc Med. 2019;20:1112-6.

9. Angelini P. Novel imaging of coronary artery anomalies to assess their prevalence, the causes of clinical symptoms, and the risk of sudden cardiac death. Circ Cardiovasc Imaging. 2014; $7: 747-54$

10. Boutsianis E, Dave H, Frauenfelder T, et al. Computationa simulation of intracoronary flow based on real coronary geometry. Eur J Cardiothorac Surg. 2004;26:248-56.

11. Theodorakakos A, Gavaises M, Andriotis A, et al. Simulation of cardiac motion on non-Newtonian, pulsating flow development in the human left anterior descending coronary artery. Phys Med Biol. 2008;53:4875-9.

12. Cho Yl, Kensey KR. Effects of the non-Newtonian viscosity of blood on flows in a diseased arterial vessel. Part 1: steady flows. Biorheology 1991:28:241-62.

13. Karino $\mathrm{T}$ Goldsmith $\mathrm{HL}$, Motomiya $\mathrm{M}$, et al. Flow patterns in vessels of simple and complex geometries. Ann NY Acad SCi. 1987:516:422-41

14. Johnston BM, Johnston PR, Corney S, Kilpatrick D. Non-Newtonian blood flow in human right coronary arteries: steady state simulations. J Biomech. 2004;37:709-20.

5. Chu M, von Birgelen C, Li Y, et al. Quantification of disturbed coronary flow by disturbed vorticity index and relation with fractional flow reserve. Atherosclerosis. 2018;273:136-44.

16. Pao YC, Lu JT, Ritman EL. Bending and twisting of an in vivo coronary artery at a bifurcation. J Biomech. 1992:3:287-9.
17. Basso C, Maron BJ, Corrado D, Thiene G. Clinical profile of congenital coronary artery anomalies with origin from the wrong aortic sinus leading to sudden death in young the wrong aortic sinus leading to sudden death in young

18. Angelini P. ACAOS is better revealed by intravascular ultrasonography than by computed tomographic angiography. Tex Heart Inst I. 2015:42:246-7.

19. Angelini P, Velasco JA, Ott D, Khoshnevis GR. Anomalous coronary artery arising from the opposite sinus: descriptive features and pathophysiologic mechanisms, as documented by intravascular ultrasonography. J Invasive Cardiol. 2003; 15:507-14

20. Rigatelli G, Roncon L, Giordan M, Cardaioli P. Symptomatic anomalous intramural coronary artery. J Cardiovasc Med (Hagerstown). 2008;9:1293-4.

21. Kaushal S, Backer CL, Popescu AR, et al. Intramural coronary length correlates with symptoms in patients with anomatous ength core 2011:92:986-91.

22. Nees SN, Flyer JN, Chelliah A, et al. Patients with anomalous aortic origin of the coronary artery remain at risk after surgical repair. J Thorac Cardiovasc Surg. 2018;155:2554-64.

23. Rigatelli $G$, Cardaioli P. Endovascular therapy for congenita coronary artery anomalies in adults. I Cardiovasc Med (Hagerstown). 2008;9:113-21.

24. Sinha SK, Razi M, Mahrotra A, et al. Anomalous coronary artery from the opposite sinus (ACAOS): technical challenges during percutaneous coronary intervention. Cardiol Res. 2018:9:120-4. 